\title{
Comparison of Saw Blade Natural Frequencies and Critical Speeds Using CSAW and Finite Element Analysis
}

\author{
J. Poirier ${ }^{1}$ P. Radziszewski ${ }^{1}$ \\ Department of Mechanical Engineering, McGill University, Montreal, QC H3A 2K6
}

\begin{abstract}
The natural frequencies of circular saws limit the operating speeds of the saws. Current industry methods of increasing natural frequency include pretensioning, where plastic deformation is induced into the saw. To better model the saw, the finite element model is compared to current software for steel saws; C-SAW, a software program that calculates frequencies for stiffened circular saws. Using C-SAW and the finite element method the results are compared and the finite element method is validated for steel saws.
\end{abstract}

\section{Introduction}

Circular saw disks used in the lumber industry are limited in operation by their critical speed. To increase the critical speed of the disk several techniques are used. Tensioning where the disk is deformed about a diameter

Guides are also extensively used to dampen vibrations and increase the stiffness of the disk, thereby increasing the critical speed of the disk.

CSAW [1] is a commercial saw-modeling program to help predict saw response based upon the parameters of the saw and any improvements applied. The software can be used in an industrial setting to better predict where to apply pretensioning to the saw blade. It is also capable of simulating many of the various guide systems and saw arbors available.

The authors' previous work has looked at the application of Shape Memory Alloys to spinning disks such as saw blades and their impact on natural frequency and critical speed. [2] To validate the FEM model, the authors have used the commercial CSAW software to compare their results.

\section{CSAW}

The CSAW software was developed in 1980 as a program to predict the natural frequencies of an rotating saw and its corresponding critical speeds. This allowed saw manufacturers and operators to better plan parameters such as operating speeds, tensioning, and improvements such as guards and guides.
Reponses of the saw can be calculated from input forces.

\section{Mechanics of Vibration of a Circular Saw}

The operating speed of the saw is limited by its critical speed. The critical speed being the speed at which the natural frequency of the saw is equal to a rotating speed of the saw as shown in equation (1). When the equation is satisfied the saw will become resonant and unstable resulting in a larger cutting width or even saw buckling or failure. The effect of heating on the saw teeth results in large compressive hoop stresses near the periphery of the disk. The compressive hoop stresses lower the stiffness of the saw, resulting in lowered natural frequencies. Tensioning is accomplished by deforming the inner part of the saw, inducing tensile forces along the periphery to counter the thermal compressive stresses.

$\omega_{\text {critical }}=\omega_{\text {rotation }} \Rightarrow \frac{\omega_{n(\text { backwards })}}{n}-\omega_{\text {rotation }}=0$

The modes of vibration consist of a forward and backwards traveling wave. It is the backwards traveling wave that is of concern, as it decreases in frequency with increased rotation speed when observed from a stationary observer. When critical, the wave will appear to be standing to the observer. The forward traveling wave will increase with increase in rotation speed. The modes of vi bration of interest are 
the low diameter, zero circumferential nodes, particularly the 2 diameter, 0 circumference node.

\section{Thermal Distribution}

The thermal distribution of the disk was determined from the method outlined in [1]. The periphery of the saw was held at a constant temperature. Heat transfer was possible by convection only along the sides of the disk and the clamping surface. There was no heat conduction along the shaft.

The diagram shows the distribution of the temperature along the disk. The temperature is uniform in the axial direction. The temperatures are relative to $0 \mathrm{deg}$ ambient temperature.

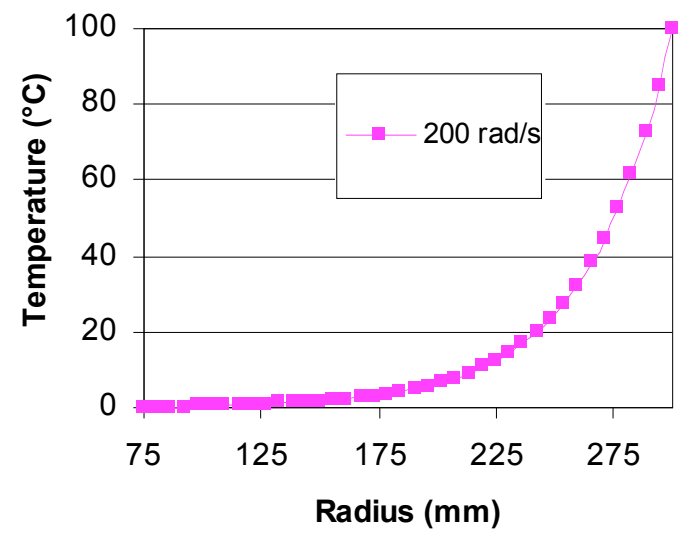

Figure 1. Temperature distribution in disk

\section{Setup}

For the simulation a simple clamped saw was investigated. The use of guides or floating arbors was not considered.

The saw modeled was $600 \mathrm{~mm}$ in diameter and 5 $\mathrm{mm}$ thick. The saw was clamped at a diameter of 150 $\mathrm{mm}$ and ran on a shaft $50 \mathrm{~mm}$ in diameter. For the finite element model, the saw was restricted in transverse motion at the clamping radius, radial motion was permitted.

The edge temperature applied to the disk varied from $50 \mathrm{deg}$. C to $150 \mathrm{deg}$. C. The rotation speed of the disk was from $100 \mathrm{rad} / \mathrm{s}$ to $300 \mathrm{rad} / \mathrm{s}$.

The disk has the following properties.
Figure 1. Temperature distribution in disk

\begin{tabular}{|c|c|}
\hline Young's Modulus & $200 \mathrm{Gpa}$ \\
\hline Density & $7850 \mathrm{~kg} / \mathrm{m} 3$ \\
\hline Poissons Ratio & 0.3 \\
\hline Expansion Coefficient & $11^{*} 10^{\wedge}-6 / \mathrm{degC}$ \\
\hline Clamp radius & $75 \mathrm{~mm}$ \\
\hline Disk radius & $300 \mathrm{~mm}$ \\
\hline Plate Thickness & $5 \mathrm{~mm}$ \\
\hline
\end{tabular}

\section{Results and Discussion}

The results of the finite element model correspond well with the results obtained from the CSAW program. These results also conform to the model used in figures 2 and 3 . The FEA model produced slightly higher natural frequencies than the CSAW model. This could be due to the clamp being rigid in the FEM model, whereas the CSAW used a thick steel clamp, slightly reducing the stiffness. This resulted in the CSAW saw becoming critical at lower temperatures than the FEA saw, $\sim 7$ to $10^{\circ} \mathrm{C}$.

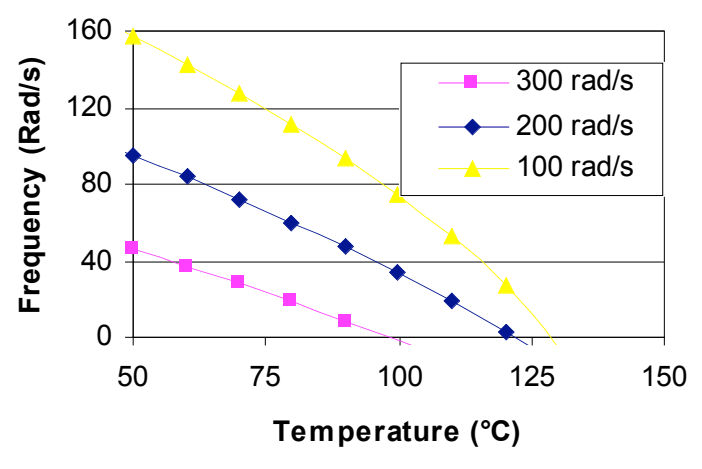

Figure 2. Frequency of 2 diameter mode of vibration from stationary observer (FEM) 


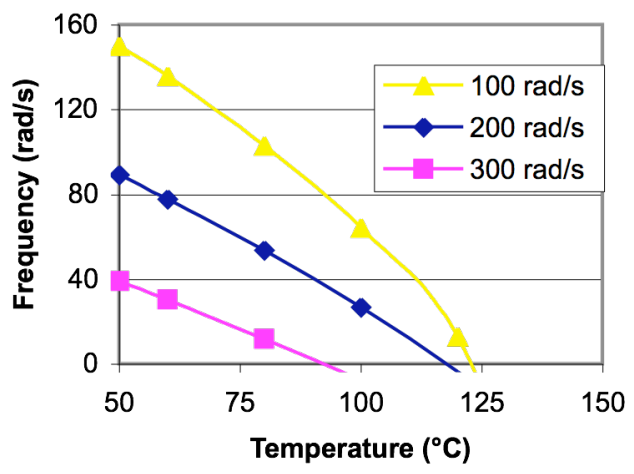

Figure 3. Frequency of 2 diameter mode of vibration from stationary observer (CSAW).

The critical speed of the disk from the finite element model was found through iteration and was not directly produced as with the CSAW.

The higher modes of vibration also corresponded well between the FEM and CSAW models as seen in figure 4 .

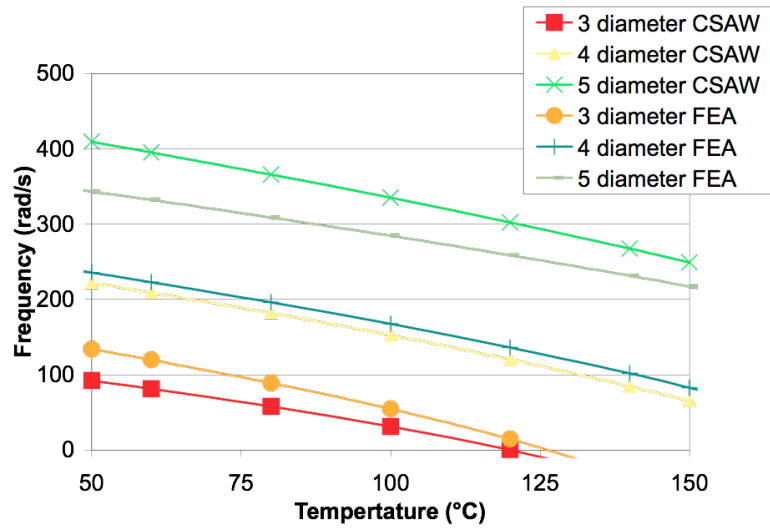

Figure 4. Frequency of 0 circumference, multiple diameter modes of vibration for rotating saw at $200 \mathrm{rad} / \mathrm{s}$.

\section{Conclusions and Future Work}

The results from CSAW did correspond with the FEM results. The CSAW produced results slightly lower than the finite element model, largely in part to the restraint condition imposed in the FEA model.

Future work will include modeling the pretensioning effect in the FEM, and comparing the results obtained to those of CSAW. And further modeling of the SMA saw and comparison to pretensioned saws.

\section{References}

[1] Dr. Gary Schajer, CSAW, Wood Machining Institute, 1985

[2] Poirier, J. Radziszewski, P. "Tensioning Circular Saws through the use of Shape Memory Alloys" Proceeding of Sawtech 2005, 2005.

[3] Mote Jr., C.D., "Theory of Thermal Natural Frequency Variations in Disks" International Journal of Engineering Science, vol. 8, 1966, pp. 547-557 